\title{
Characterization of Genes Involved in $\gamma$-Aminobutyric Acid Metabolic Pathways Response to Metabolites Accumulation in Embryos during Barley Germination
}

\author{
Mengyuan Jin ${ }^{1}$, Qin Wu ${ }^{1}$, Peiwen Mao', Zhiwei Zhou ${ }^{1,3}$, Weiran Cong ${ }^{2}$, Juan Lin ${ }^{3}$ and Xuanwei Zhou ${ }^{1 *}$ \\ ${ }^{1}$ School of Agriculture and Biology, Engineering Research Center of Cell \& Therapeutic Antibody, Ministry of Education, \\ Shanghai Jiao Tong University, Shanghai 200240, People's Republic of China \\ ${ }^{2}$ Xuncaofang (Shenyang) Biological Technology Co., Ltd., Benxi 117004, People's Republic of China \\ ${ }^{3}$ School of Life Sciences, Fudan University, Shanghai 200439, People's Republic of China \\ *For Correspondence: xuanweizhou@sjtu.edu.cn; xuanweizhou@163.com \\ Received 13 May 2020; Accepted 04 January 2021; Published 25 March 2021
}

\begin{abstract}
To reveal the key enzyme genes involved in $\gamma$-aminobutyric acid (GABA) metabolic pathways response to elevated metabolite storage in embryos during barley germination, this study investigated the GABA content, cloned GABA metabolic pathway genes and analyzed their expression levels, respectively. In barley embryos, GABA content continued to rise during the soaking process and then decreased after the germination. Three genes including glutamic acid decarboxylase (GAD), GABA transaminase (GABA-T) and succinic semialdehyde dehydrogenase (SSADH) involved in the GABA pathway were cloned and characterized from the barley embryos, respectively. Before the germination, the expression of GAD gene was upregulated, while GABA-T gene expression was down-regulated. After the germination, GAD gene expression was lowered, but GABA-T gene expression was rapidly increased. The SSADH gene expression remained stable after soaking of $4 \mathrm{~h}$, and then down-regulated. There is evidence that the high GABA content in germinating barley seeds is parallel with the upregulation of the GAD gene, and down-regulation of GABA-T gene. These results indicate that the expression level of the genes involved in GABA pathway is a crucial factor in GABA accumulation during soaking and germination. This study is beneficial for the development of GABA-rich barley products by germination. (C) 2021 Friends Science Publishers
\end{abstract}

Keywords: Barley grains; Germination; Embryos; $\gamma$-aminobutyric acid; Gene expression

\section{Introduction}

Gamma-aminobutyric acid (GABA) is a four-carbon nonprotein amino acid isolated from potato tubers in plants (Steward 1949). It has a variety of bioactivity such as inhibitory neurotransmitter (Carmans et al. 2013), lower blood pressure (Kajimoto et al. 2004), replenish the brain, treat mental illness, and improve immunity (Zhang et al. 2002; Diana et al. 2014). Because of the multiple effects on human health, the development of GABA-rich products has attracted widespread interest among researchers. In Japan, GABA-rich tea, Gabaron, was brought to the market as a functional food with blood pressure lowering effect (Wang et al. 2013). GABA has been identified as a new resource food, and extensively used in foods such as soft drinks, condiments, and dairy products by the Chinese Ministry of Health (Liang et al. 2013). However, the GABA content is maintained at a low level in an organism. The content ranges of $0.03 \sim 2.58 \mathrm{mg} / \mathrm{g}(\mathrm{FW})$ in plant tissues under normal growth conditions (Shelp 2012). For example, the
GABA content is about $0.06 \sim 0.09 \mathrm{mg} / \mathrm{g}$ (DW) in brown rice, lower in refined rice (Zhang et al. 2002). Germination is an effective process for improving the nutritional quality and functionality of cereals. The content of GABA in germinated grains was higher than in the un-germinated grains (Gangopadhyay et al. 2015). Therefore, developing GABA-rich cereal food with lowering blood pressure function can largely and effectively alleviate the symptoms of hypertension in many individuals and reduce the mental stress and economic burden in the hypertensive population.

Accumulation of GABA content in plant is related to its anabolic and catabolism pathways. Metabolic pathway of GABA is a metabolic bypass of tricarboxylic acid (TCA) in the plant that start with TCA cycle in which $\alpha$-ketoglutarate is catalyzed by glutamate dehydrogenase (GHD) to produce glutamate (Glu) (Ling et al. 1994; Schultz and Coruzzi 1995). When Glu is transported from mitochondria across the mitochondrial membranes into the cytoplasm, it is catalyzed by glutamate decarboxylase (GAD) to generate GABA through an irreversible reaction, and then 
transported into mitochondria. GABA produces the succinic semialdehyde (SSA) through GABA-T action (Shelp et al. 1999; Van Cauwenberghe and Shelp 1999). Succinic semialdehyde dehydrogenase (SSADH) catalyzes a reaction through SSA to generate succinate involved in the TCA cycle. Furthermore, the reaction catalyzed by SSADH is an irreversible reaction (Bouche and Fromm 2004; Akçay et al. 2012). Otherwise, SSA generates a $\gamma$-hydroxybutyrate (GHB) (Fig. 1). According to which, the synthesis of GABA by activating GAD or inhibiting GABA-T or SSADH enzyme activity has become a research hotspot. GAD is one of the first key enzymes during the germination of plant seeds (Lamkin et al. 1983). GAD enzyme activity in germination of soybean seed is correlated with the content of GABA (Xu and $\mathrm{Hu} 2014$ ). GAD enzyme activity after soaking in rice is positively correlated with the accumulation of GABA, which indicate that the accumulation of GABA in embryo after soaking is related to the catalytic reaction of GAD (Liu et al. 2005). GABA-T is a key enzyme in the GABA degradation pathway. In previous studies, such as rice and tomato using RNA interference technique to decrease the expression of $G A B A$ $T$ can increase GABA content (Koike et al. 2013; Zhou et al. 2015). Therefore, it is very important to regulate the gene expression of GABA synthesis and degradation pathway to obtain a rich-GABA food.

Hordeum vulgare Linn. var. nudum Hook. f., mainly distributed in the Qinghai-Tibet Plateau, is a typical nutrition-balanced crop, with high content of dietary fiber, vitamins and mineral elements, moderate protein, and low sugar and fat (Lin et al. 2016). In addition, barley grains are also rich in GABA, $\beta$-glucan and polyphenols, etc. (Yang et al. 2015). The rich bioactive ingredient makes it become a valuable raw material for the development of functional food (Zhou et al. 2018). However, the studies were based on barley grains, but the accumulation of GABA was mainly occurred in embryo and its molecular mechanisms were not reported. In the present study, first time, the changes of GABA content in embryos of the barley grains were analyzed during the whole germination process, then the relative gene involved in GABA metabolisms pathway were cloned, while the gene expression of GABA metabolic pathway during the soaking and germination were investigated. Results will provide a theoretical basis for the development of germinating products and the molecular breeding of barley grains through studying of the accumulation of GABA and their molecular regulation mechanism during the germination.

\section{Materials and Methods}

\section{Seeds soaking, germination and embryos collection}

The barley grains were provided by Tibet Shenglong Industry Co., Ltd. The barley seeds, were soaked and germinating barley seeds were used further as previously described (Zhang and Zhou 2014), subsequently the embryos were isolated carefully from barley seed using a scalpel and forceps, which frozen in liquid nitrogen for further use.

\section{Extraction and determination of GABA}

For this purpose, principal method was adapted as reported earlier (Liu et al. 2018). The collected embryos sample was inactivated at $85^{\circ} \mathrm{C}$ for $30 \mathrm{~min}$, dried at $65^{\circ} \mathrm{C}$ to constant weight, and then grounded into powder. The powder $40 \mathrm{mg}$ and $5 \mathrm{~mL}$ distilled water were added into a $15 \mathrm{~mL}$ centrifuge tube, treated for $4 \mathrm{~h}$ at $200 \mathrm{rpm}$ and $60^{\circ} \mathrm{C}$ on a constant temperature oscillator, cycled for three times, and collected the extracting solution. The extracting solution was mixed together, and then centrifuged at $4000 \mathrm{rpm}$ for $10 \mathrm{~min}$, and returned to the supernatant for determination of GABA. The GABA content from the extraction was carried out by the colorimetric method (Luo et al. 2014) at $645 \mathrm{~nm}$ wavelength.

\section{RNA extraction}

Total RNA was extracted using RNA pure Plant Kit (CWBIO, Beijing, China). The quality and concentration of isolated RNA were checked by agarose gel electrophoresis using a spectrophotometer (WFZUV-2100, Unico ${ }^{\mathrm{TM}}$ Instruments Inc.). Total RNA was treated with RNase-free DNAase I (Promega, Madison, W.I., U.S.A.) to remove contaminating genomic DNA.

\section{Cloning of GABA branch genes and bioinformatic analysis}

A Takara reverse transcription PCR kit was used for synthesis of first-strand cDNA from about $1 \mu \mathrm{g}$ RNA. According to three putative GABA branch gene sequences searched from the National Center for Biotechnology Information (NCBI) (AK355055, AK249113, AK248458), primers listed in Table 1 were designed using Primer Premier 5.0 software (Premier Biosoft International, Palo Alto, C.A., U.S.A.). After PCR and following A-tailing procedure, the purified target fragment was inserted into a pMD 19-T Vector (Takara, Bio Inc., Shiga, Japan) and transferred into E. coli DH-5 $\alpha$ (Vazyme Biotech Co., Ltd., Nanjing, China). Positive clones were sequenced by Shanghai Ruidi Biotech Company (Shanghai, China).

\section{Quantitative reverse transcription-PCR (qRT-PCR)}

The relative gene expression analysis was performed with Thermo real-time PCR (RT-PCR) for 8 embryos from different soaking and germination periods of barley grains. Total RNA was extracted using a CWBIO kit (Beijing China) and cDNA synthesis was performed by reverse transcription kit (Takara, Dalian, China) according to the 
Table 1: Primers used in this study

\begin{tabular}{lll}
\hline Names & Sequences & Target fragment \\
\hline Primers used in the gene cloning & 5'-CAGAGCCAAGAGCGAGTAGC-3' & $1549 \mathrm{bp}$ \\
GAD-F & 5'-GCTTGGATTTTGGACGCTG-3' & $1631 \mathrm{bp}$ \\
GAD-R & 5'-ATGACGATGATTGCCCGCGGC-3' & $1595 \mathrm{bp}$ \\
GABAT-F & 5'-CACGGTGTTATTACTGGCATTTG-3' \\
GABAT-R & 5'-ATGGGCAGCGTGGACGCGG-3' \\
SSADH-F & 5'-CCATCTTACACCTTACGCCT-3' & $170 \mathrm{bp}$ \\
SSADH-R & 5'-TCGACATCGACACCGTCATGG-3' \\
Primers used in the qRT-PCR & 5'-CTTCTTGGCGAGCACAAACTC-3' \\
qGAD-F & 5'-ACGTGGCCTGGGATTGATAC-3' & $138 \mathrm{bp}$ \\
qGAD-R & 5'-CCTGCGACCCTGATAAGCAT-3' & $137 \mathrm{bp}$ \\
qGABAT-F & 5'-AGCCAACTGTGGTAGGGAAC-3' \\
qGABAT-R & 5'-TAAGCCTGCATTGGTGTCGT-3' & $139 \mathrm{bp}$ \\
qSSADH-F & 5'-CCAGGTATCGCTGACCGTAT-3' & \\
qactin-F & 5'-GGAAAGTGCTGAGTGAGGCT-3' & \\
qactin-R & & \\
\hline
\end{tabular}

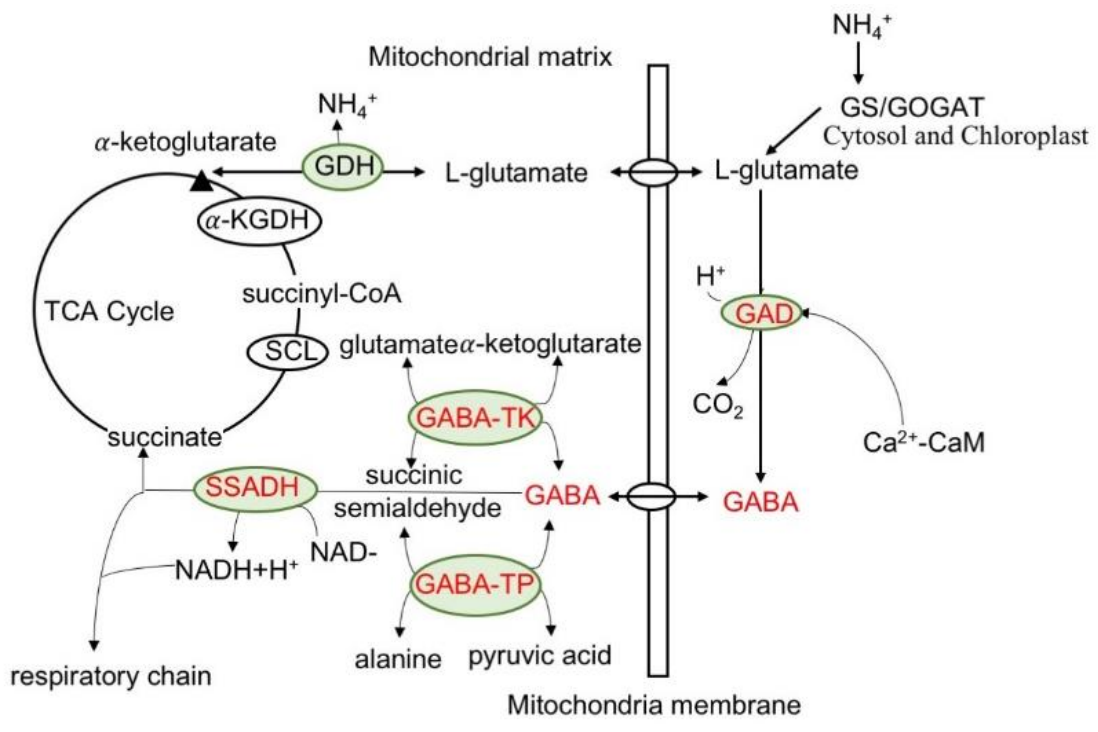

GABA: gamma aminobutyric acid

SCL: succinyl CoA ligase

a-KGDH: $\boldsymbol{\alpha}$-ketoglutarate dehydrogenase

GDH: glutamate dehydrogenase
GAD: glutamate decarboxylase

GABA-TK (P): GABA transaminases

using a-ketoglutarate or pyruvate

SSADH: succinate semialdehyde dehydrogenase

GS/GOGAT: glutamine synthetize/glutamate synthase

Fig. 1: GABA metabolic pathway and its regulation in plant

manufacturer's instructions. Then qRT-PCR was carried out with Takara SYBR Kit (TaKaRa, Dalian, China) in accordance with the manufacturer's protocols. Using $\beta$ actin (GeneBank: AY145451.1) from barley as internal reference gene, the specific primers for qRT-PCR were designed according to reference mRNA sequences of GAD, GABAT, SSADH by using NCBI's pick primer software. The program was set as $30 \mathrm{~s}$ at $95^{\circ} \mathrm{C}, 40$ cycles for $5 \mathrm{~s}$ at $95^{\circ} \mathrm{C}$ and $30 \mathrm{~s}$ at $60^{\circ} \mathrm{C}$, with a default melting curve stage for $15 \mathrm{~s}$ at $95^{\circ} \mathrm{C}, 1 \mathrm{~min}$ at $60^{\circ} \mathrm{C}$ and $15 \mathrm{~s}$ at $95^{\circ} \mathrm{C}$. The target gene expression change was calculated by the comparative $\Delta \mathrm{Ct}$ method regarding barley $\beta$-actin gene as an internal control. All the experiments were conducted for three times repetitions and the primers were listed in Table 1.

\section{Statistical analysis}

All experiments contained three parallel tests. Calculations of mean, standard deviation (SD), and $P$-values were performed on triplicate experiments using S.P.S.S. 19.0 software (S.P.S.S. Inc., N.Y., U.S.A.). The Student's t test 


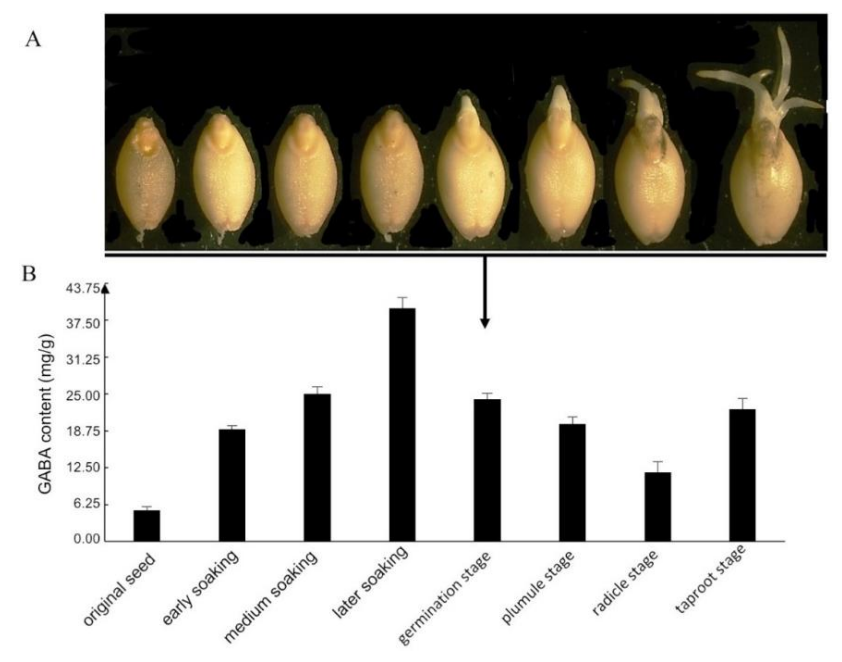

Fig. 2: Changes in GABA content during barley grains germination

A. The process of barley grains germination; B. GABA content in embryos from different periods of barley grains germination

was used to calculate $P$-values for comparison. Significant statistics were set at a $P$-value $<0.05$.

\section{Results}

\section{Collection of samples}

The barley embryos were selected from the soaking and germination periods. The soaking period included original seed, early ( 2 h), middle (4 h) and later (6 h) soaking, and the germination period included germination, plumule, radicle and taproot stages (Fig. 2A). Samples also represented morphological characters of barley seed during soaking and germination.

\section{Changes of GABA content in embryos}

Base on the established standard curves of GABA, the regression $\mathrm{R}^{2}=0.99$ showed that there exists a linear relationship between GABA content and the corresponding absorption wave when the GABA content varied range from 0 to $0.5 \mathrm{mg}$, so it can be used to determine the GABA content in the sample. The results showed that the GABA content continued to rise during soaking. For soaking duration of 6 h, GABA content was the highest $(40 \mathrm{mg} / \mathrm{g})$, about 8 times than in untreated dry seed embryos. However, the GABA content decreased after germination (Fig. 2B).

\section{Cloning and sequence analysis of GABA branch gene}

Based on the information related to GABA pathway such as GAD, GABA-T and SSADH, three genes have been isolated from barley grains by using PCR techniques (Fig. 3A-C). The GAD sequence obtained by the open reading frame

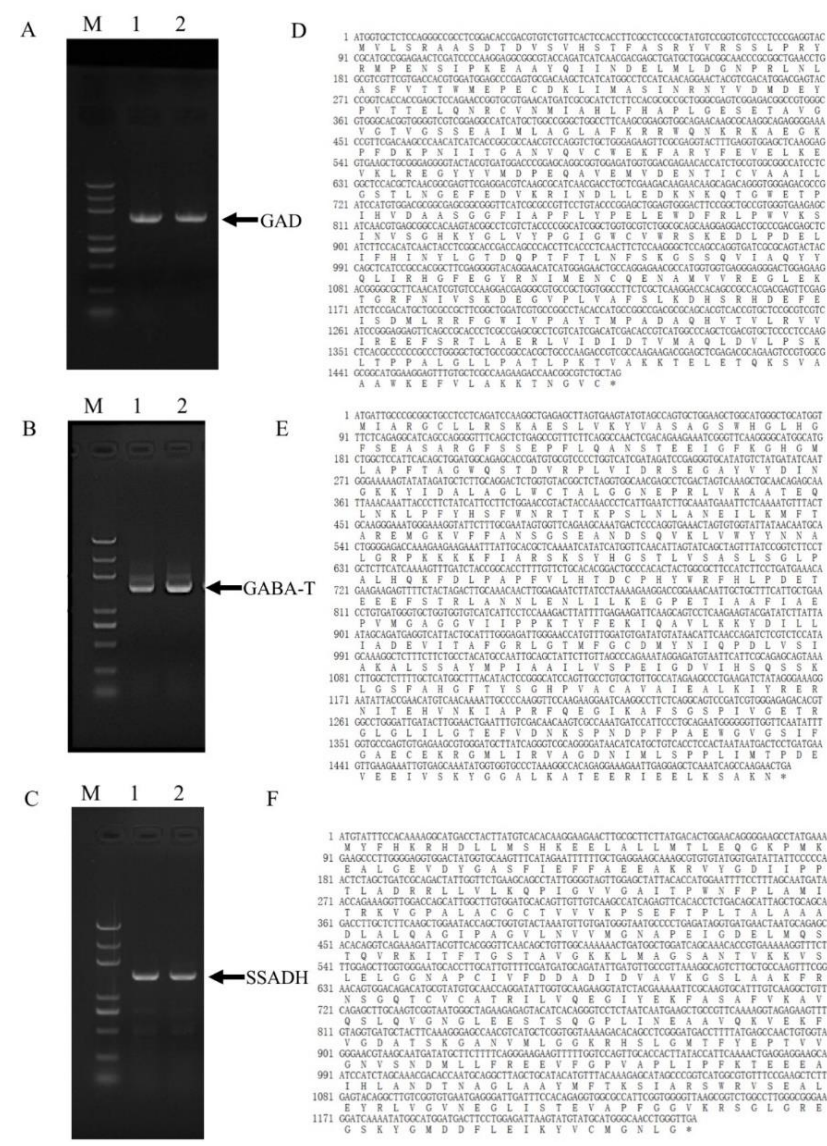

Fig. 3: The cloning of key gene of GABA pathway

A, B, C. Electrophoretogram of GAD, GABA-T and SSADH cDNA PCR products, respectively; D, E, F. Open reading framework of GAD, GABA-T and SSADH gene sequence and the corresponding amino acids sequence, respectively

(ORF)-Finder of NCBI, harbored a 1491 bp ORF that encoded 496 amino acids (Fig. 3D). The GAD sequence has the highest similarity with Aegilops tauschii GAD1-like (XM_020292464.1), which is 95\% using BLASTN analysis. And the sequence similarity of other gramineous plant, such as Brachypodium distachyon GAD1 (XM_003558361.3), Oryza sativa GAD1 (XM_015772426.1), Setaria italic GAD1 (XM_004985025.2), Sorghum bicolor GAD1 (XM_002468163.2), Zea mays GAD1 (NM_001174470.1) is among 90-92\% (Fig. 4A). All these conclusions showed that the $G A D$ gene has been cloned successfully from barley grains. The sequence of the GABA-T positive clone analyzed by the ORF-Finder of NCBI, harbored a $1524 \mathrm{bp}$ ORF and encoded 507 amino acids (Fig. 3E). The sequence of GABA-T had similarity with A. tauschii GABA-T3 (XM_020291859.1), which is 95\% using BLASTN analysis. And the sequence similarity of other gramineous plant, such as B. distachyon GABA-T3 (XM_010236148.3), O. sativa GABA-T3 (XM_015792818.1), S. bicolor GABA-T3 (XM_002445162.2), S. italic GABA-T3 (XM_004972552.3), Z. mays GABA-T1 (XM_008670668.2) is among $81-90 \%$ (Fig. 4B). The sequence of the SSADH positive clone analyzed by the ORF-Finder of NCBI, 
A

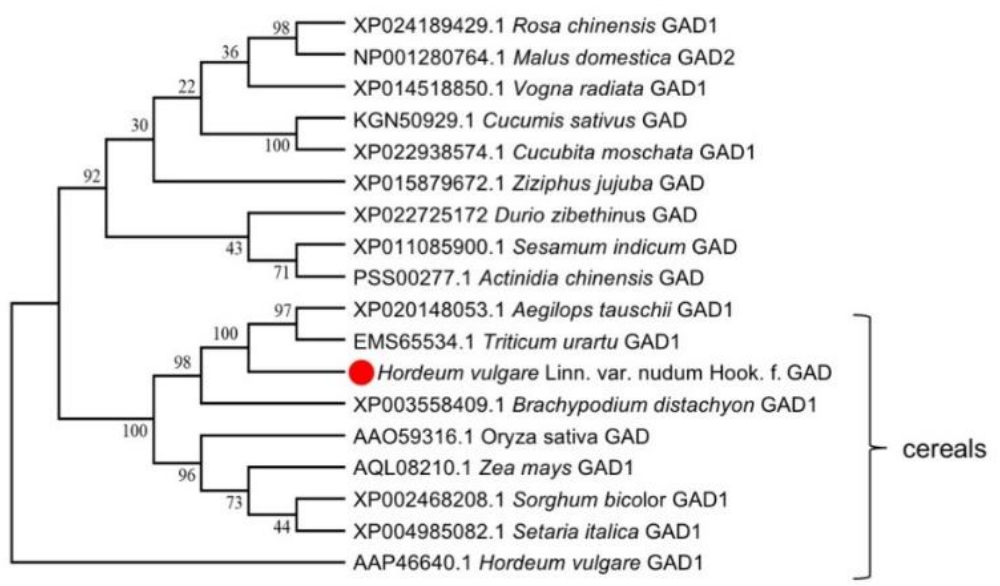

B

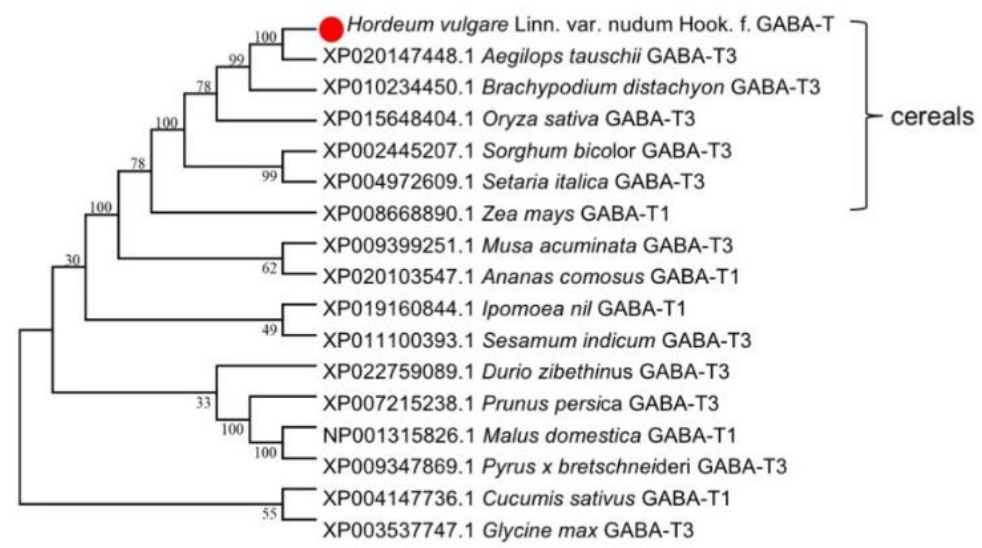

$\mathrm{C}$

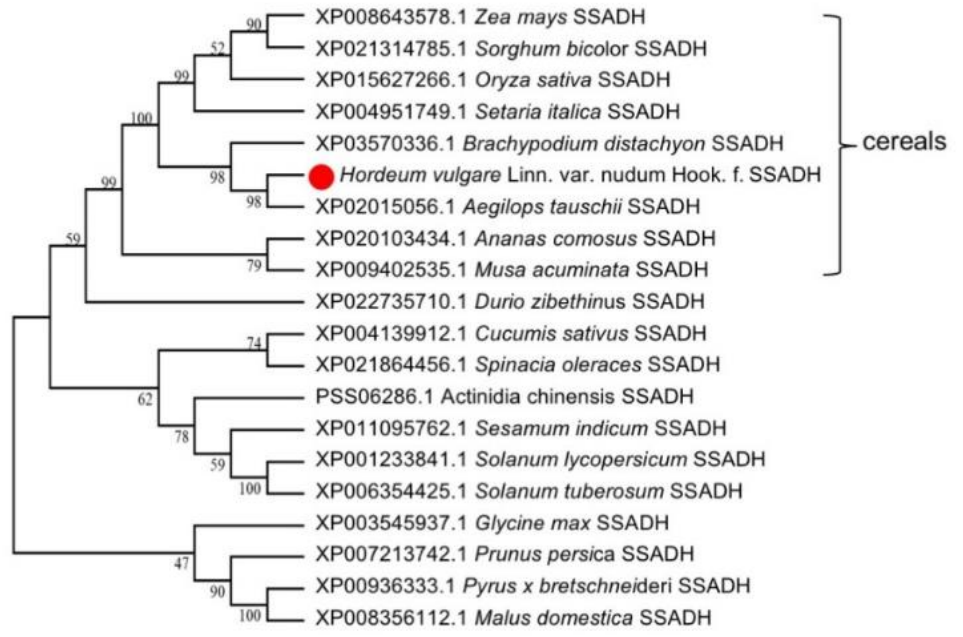

Fig. 4: Phylogenetic tree of key gene of GABA pathway

A, B, C. Phylogenetic tree of GAD, GABA-T and SSADH amino acids sequence, respectively

harbored a $1236 \mathrm{bp}$ ORF and encoded 411 amino acids (Fig. $3 \mathrm{~F})$. The sequence of SSADH was analyzed by BLASTN, and the results showed that the sequence similarity is the highest with A. tauschii SSADH (XM_020294976.1), which is $96 \%$. And the sequence similarity of other gramineous plant, such as B. distachyon SSADH (XM_003570288.4), S. bicolor SSADH (XM_021459110.1), S. italic SSADH (XM_004951692.4), Z. mays SSADH (NM_001153701.1), O. sativa SSADH (XM_015771780.1), is among 88-89\% (Fig. 4C). And these conclusions showed that the GAD, GABA-T, SSADH gene of barley grains has been cloned successfully. 
A

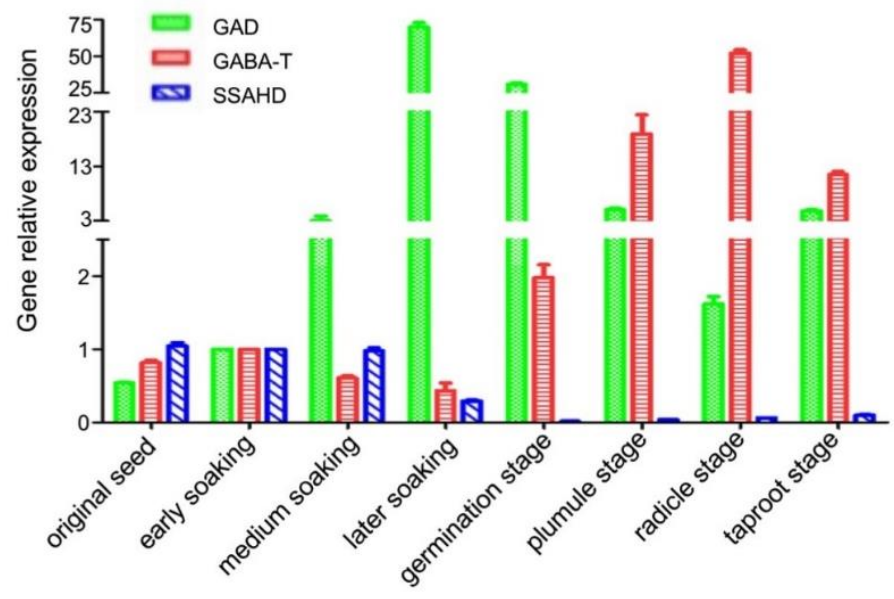

B

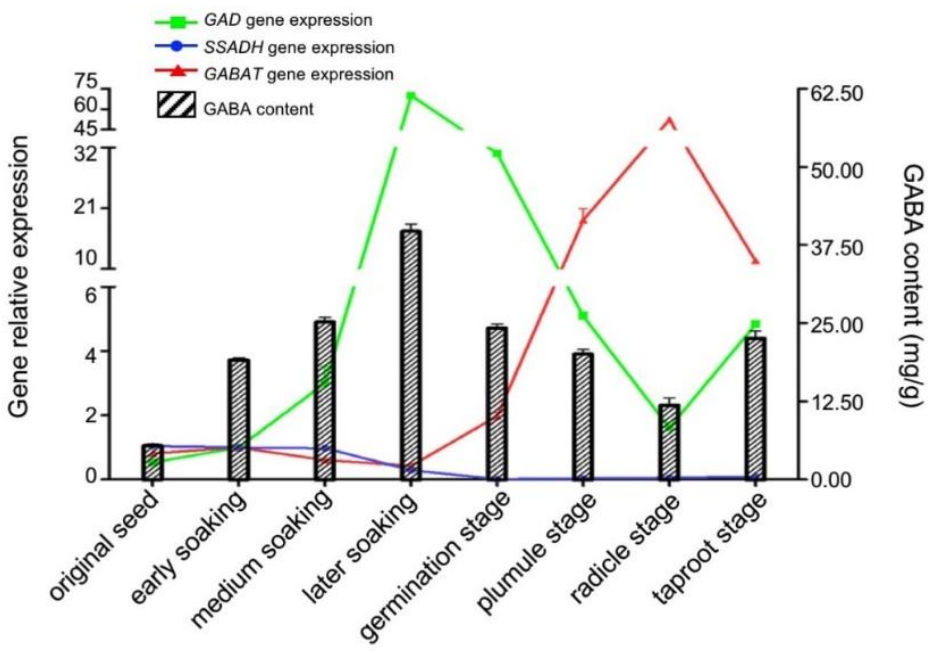

Fig. 5: The Relative expression of key gene in GABA pathway and the comparison of GABA content during grains germination A. Relative expression of key gene in GABA pathway in barley grains embryo during germination; B. Comparison of gene expression in GABA pathway and GABA content during germination of barley grains

\section{Expression analysis of GABA branch gene in soaking and germination}

Expression of three genes involved in GABA pathway in the embryo during germination of barley grains was determined using qRT-PCR. The results showed that the expression of $G A D$ showed an upward trend before the bud germination period and decreased after germination (Fig. $5 \mathrm{~A})$. However, the expression of $G A B A-T$ was downregulated before the bud sprouting period, and then increased rapidly. The expression of SSADH mainly stayed stable but deceased after soaking for $4 \mathrm{~h}$. From the early stage to the late stage during soaking (from $2 \mathrm{~h}$ to $6 \mathrm{~h}$ ), the expression of $G A D$ genes which controlled GABA synthesis was significantly up-regulated, while the expression of $G A B A-T$ and $S S A D H$ in the GABA degradation pathway showed a downward trend (Fig. 5B). Thus, it was speculated that the continuous increase of GABA content during this process may be associated with changes in gene expression. From germination to rooting, the $G A D$ gene expression decreased rapidly, while the $G A B A-T$ gene expression which controlled the first step of the GABA degradation pathway increased promptly, and is speculated to cause GABA content to decrease. After rooting, the expression of $G A D$ in multiple root stage was higher than its expression in single root stage. Besides, the expression of GABA-T in multiple root stage became lower, and the GABA content also showed an upward trend after rooting. The results also showed that during the entire germination treatment, the expression of the SSADH remained basically unchanged, but then its expression was down-regulated after soaking for $4 \mathrm{~h}$.

\section{Discussion}

Changes in GABA content were accordant with the 
expression level of gene and the key enzyme activity in metabolic pathway in various tissues and at different stages of plant development. For example, there's a lot of GABA in the fruit before the discoloration period of tomato and citrus fruit, then the GABA is quickly broken down. This is highly correlated with the activity of the synthase and catabolism enzyme in the GABA pathway of tomato fruit (Diaz et al. 2005; Cercos et al. 2006; Akihiro et al. 2008). Metabolic pathway of GABA consists of three enzymes: GAD, GABA-T and SSADH. Therefore, it is important to colon the key enzyme genes for analysis of GABA accumulation in germination at the molecular level. The seed embryo is the storage place of GABA in barley grains (Inatomi and Slaughter 1971), therefore, embryo was selected for further study.

It was demonstrated that changes in the gene expression whose mRNA levels and their encoding enzymes are related to GABA content in the various tissues. The GABA content is determined by the key enzyme gene both the synthesis and the decomposition pathways. The $G A D$ gene is generally considered to be the key enzyme gene in the GABA synthesis pathway, and the increase of GAD enzyme activity is the main factor leading to GABA accumulation (Baum et al. 1996; Akama and Takaiwa 2007; Hyun et al. 2013; Xu and $\mathrm{Hu} 2014$ ). For example, studies in soybeans have shown that the GAD enzyme activity increases with germination, and that the mRNA expression level of GAD maintains a relatively high level 15 to 35 days after flowering (Clark et al. 2009; Takahashi et al. 2013; Xu and $\mathrm{Hu} 2014$ ). Prior to the maturity of tomato fruit, GAD, a key enzyme activity in the GABA synthesis pathway is high. This indicates that the GABA accumulation is positively correlated with the activity of GAD enzyme. Decreased the downward trend of GABA-T and SSADH enzyme activity in the GABA decomposition pathways can also lead to GABA accumulation. For example, GABA is rapidly degraded after the ripening stage of tomato fruit. In the same time, the expression of S1GABA-T1 is greatly increased. This indicates that GABA-T plays a key role in the GABA decomposition pathways (Akihiro et al. 2008; Koike et al. 2013). However, some studies have suggested that there is no significant relationship between changes in the key genes expression of the GABA pathway and GABA content. For example, the GABA content of the frost-resistant barley grains increased 15-fold in response to freeze stress, but without accompanying changes in the expression of $G A D$, GABA-T and SSADH genes (Mazzucotelli et al. 2006). It was found that the changes of GABA concentration did not accompany any change of $G A B A-T$ transcriptional abundance when Arabidopsis was domesticated at low temperature (Kaplan et al. 2007), while it was also found to have no obvious relationship between the change of $G A B A$ $T$ and GABA content rice grains (Narsai et al. 2009). In the present study, GABA content in the barley seeds increased gradually with soaking and up to the highest at $6 \mathrm{~h}$ but decreased after germination. Correspondingly, the expression of GAD gene continued to increase after immersion in water, and reached the highest point in the later period of water swelling (soaking for $6 \mathrm{~h}$ ), this indicates that the increase of GABA content is indeed related to the activation of GAD gene. From the principle of gene expression regulation products, the increase in gene expression should be earlier than the increase in expression products. These experiments showed that the amount of GABA in the barley grains increased greatly in the early stage of water absorption and swelling. But there was no significant increase in gene expression, which might be related to the increasing GABA content in the embryo at the initial stage of soaking. At this stage, the increase of GABA content is due to the release of bound GABA, rather than the GAD enzymatic response (Liu et al. 2005). The enzymatic response of GAD promotes the increase of GABA content mainly in the middle and late stages of grain swelling.

These experimental results showed that, in the later stages of hydration swelling of barley grains, not only the $G A D$ expression reached the highest level, but also the amount of $G A B A-T$ and $S S A D H$ reached the lowest. Therefore, it is believed that the barley grains are dominated by GABA synthesis in the water-swelling period. After the grains became white, the GABA content began to slowly decline, but reached the lowest level in the single root stage, and then there is a slight rise, and this is consistent with the increasing trend of brown rice after germination (Zheng 2006). During the period from whitening to single root, the expression of $G A D$ gradually decreased, and the expression of $G A B A-T$ gradually increases. Therefore, the decrease of GABA expression results from the decrease in the synthesis and the increase in decomposition. The increase in decomposition is mainly due to the increase in GABA-T enzyme activity, which is consistent with studies on soybean seed (Takahashi et al. 2013). Therefore, in order to maintain the amount of GABA in the barley grain embryo, increasing GAD enzyme activity (after barley grain is becoming white) or using the GABA-T inhibitor to inhibit the expression of GABA-T should be tried to use in processing practice. The SSADH is the last key enzyme in the GABA decomposition pathway, but it does not show an increase in expression during the period from whitening to a single root. It is speculated that the decomposition of GABA in grain is mainly regulated by GABA-T. In addition, SSADH gene in plant has multiple copies, such as three copies in Arabidopsis and maize, and the numbers of SSADH copies in the barley grains are unknown, and there may be other copies of genes that contribute to GABA degradation during this period.

\section{Conclusion}

The evidence indicated that the expression level of the genes involved in GABA pathway is a crucial factor in soaking and germination. The GABA content of barley grains in the treatment process varied with the related gene changes in 
gene expression in embryos. It revealed a role that GABA is dominated by anabolic metabolism in soaking, but catabolism in germination. The investigation is beneficial for the development of $\gamma$-aminobutyric acid-rich barley products.

\section{Acknowledgments}

We would like to thank director Xin Wang from Tibet Shenglong Industry Co., Ltd for helping us to collect various samples of barley seeds. This research is financially supported by the Tibet Anada Biomedical Technology Co. Ltd. (No. 20H100000595), and the Xuncaofang (Shenyang) Biological Technology Co., Ltd. (No. 20H100000125).

\section{Author Contributions}

$\mathrm{X}-\mathrm{W} \mathrm{Z}$ managed the project; X-W Z and J L designed the experiments and provided support for the experiments; M-Y J, QW and P-W M managed the samples and performed the experiments. Z-W Z and W-R C led the data analysis and preparation of the graph, M-Y J and Q $\mathrm{W}$ drafted the manuscript; all authors reviewed and approved the manuscript.

\section{Conflict of Interest}

There is no conflict of interest among the authors and the institutions where the research has been conducted

\section{Data Availability Declaration}

All data related to this article are in the custody of corresponding author and will be available on request

\section{References}

Akama K, F Takaiwa (2007). C-terminal extension of rice glutamate decarboxylase (OsGAD2) functions as an autoinhibitory domain and overexpression of a truncated mutant results in the accumulation of extremely high levels of GABA in plant cells. $J$ Exp Bot 58:2699-2707

Akçay N, M Bor, T Karabudak, F Özdemir, İ Türkan (2012). Contribution of Gamma amino butyric acid (GABA) to salt stress responses of Nicotiana sylvestris CMSII mutant and wild type. J Plant Physiol 169:452-458

Akihiro T, S Koike, R Tani, T Tominaga, S Watanabe, Y Iijima, K Aoki, D Shibata, H Ashihara, C Matsukura, K Akama, T Fujimura, H Ezura (2008). Biochemical mechanism on GABA accumulation during fruit development in tomato. Plant Cell Physiol 49:1378-1389

Baum G, S Lev-Yadun, Y Fridmann, T Arazi, H Katsnelson, M Zik, H Fromm (1996). Calmodulin binding to glutamate decarboxylase is required for regulation of glutamate and GABA metabolism and normal development in plants. Embo J 15:2988-2996

Bouche N, H Fromm (2004). GABA in plants: Just a metabolite? Trends Plant Sci 9:110-115

Carmans S, JA Hendriks, H Slaets, K Thewissen, P Stinissen, JM Rigo, N Hellings (2013). Systemic treatment with the inhibitory neurotransmitter gamma-aminobutyric acid aggravates experimental autoimmune encephalomyelitis by affecting proinflammatory immune responses. J Neuroimmunol 255:45-53
Cercos M, G Soler, DJ Iglesias, J Gadea, J Forment, M Talón (2006). Global analysis of gene expression during development and ripening of citrus fruit flesh. A proposed mechanism for citric acid utilization. Plant Mol Biol 62:513-527

Clark SM, R Di Leo, ORV Cauwenberghe, RT Mullen, BJ Shelp (2009). Subcellular localization and expression of multiple tomato $\gamma$ aminobutyrate transaminases that utilize both pyruvate and glyoxylate. J Exp Bot 60:3255-3267

Diana M, J Quílez, M Rafecas (2014). Gamma-aminobutyric acid as a bioactive compound in foods: A review. J Funct Foods 10:407-420

Diaz C, S Purdy, A Christ, JF Morot-Gaudry, A Wingler, C MasclauxDaubresse (2005). Characterization of markers to determine the extent and variability of leaf senescence in Arabidopsis. Plant Physiol 138:898-908

Gangopadhyay N, MB Hossain, DK Rai, NP Bruntom (2015). A review of extraction and analysis of bioactives in oat and barley and scope for use of novel food processing technologies. Molecules 20:10884-10909

Hyun TK, SH Eom, YC Jeun, SH Han, JS Kim (2013). Identification of glutamate decarboxylases as a $\gamma$-aminobutyric acid (GABA) biosynthetic enzyme in soybean. Indus Crop Prod 49:864-870

Inatomi K, J Slaughter (1971). The role of glutamate decarboxylase and $\gamma$ aminobutyric acid in germinating barley. $J$ Exp Bot 22:561-571

Kajimoto O, H Hirata, S Nakagawa, Y Kajimoto, K Hayakawa, M Kimura (2004). Hypotensive effect of fermented milk containing gammaaminobutyric acid (GABA) in subjects with high normal blood pressure. J Jpn Soc Food Sci 51:79-86

Kaplan F, J Kopka, DY Sung, W Zhao, M Popp, R Porat, CL Guy (2007). Transcript and metabolite profiling during cold acclimation of Arabidopsis reveals an intricate relationship of cold-regulated gene expression with modifications in metabolite content. Plant $J$ 50:967-981

Koike S, C Matsukura, M Takayama, E Asamizu, H Ezura (2013). Suppression of $\gamma$-aminobutyric acid (GABA) transaminases induces prominent Solanum lycopersicum GABA accumulation, dwarfism and infertility in the tomato (L.). Plant Cell Physiol 54:793-807

Lamkin W, SW Nelson, BS Millet, Y Pomeranz (1983). Glutamic acid decarboxylase activity as a measure of percent germination for barley. Cer Chem 60:166-171

Liang HY, LK Deng, HL Lin, S Li D Zhang (2013). Research progress of new resource food--- $\gamma$-aminobutanoic acid(GABA). Food Res Dev 15:119-123

Lin J, TH Zhou, L Zhang, JN Meng (2016). Analysis of nutrient ingredients and physiological active substances in black and white hullessbarley from Shannan Longzi in Tibet. Food Sci Technol 41:88-92

Ling V, WA Snedden, BJ Shelp, SM Assmann (1994). Analysis of a soluble calmodulin binding protein from fava bean roots: Identification of glutamate decarboxylase as a calmodulin-activated enzyme. Plant Cell 6:1135-1143

Liu D, P Wang, ZW Zhou, XW Zhou (2018). Influence of auxiliary ingredients on the content of $\gamma$-aminobutyric acid during fermentation of highland barley by monascus. Sci Technol Cereals Oils Foods 26:68-72

Liu L, H Zhai, JM Wan (2005). Accumulation of $\gamma$-aminobutyric acid in giant-embryo rice grain in relation to glutamate decarboxylase activity and its gene expression during water soaking. Cereal Chem 82:191-196

Luo XJ, LL Guo, XY Yang, XC Liu, CL Yu, GC Qi, ZY Feng (2014). QTL mapping of $\gamma$-aminobutyric acid content in grains of barley. Southwest Chin J Agric Sci 27:950-954

Mazzucotelli E, A Tartari, L Cattivelli, G Forlani (2006). Metabolism of $\gamma$ aminobutyric acid during cold acclimation and freezing and its relationship to frost tolerance in barley and wheat. J Exp Bot 57:3755-3766

Narsai R, KA Howell, A Carroll, A Ivanova, AH Millar, J Whelan (2009). Defining core metabolic and transcriptomic responses to oxygen availability in rice embryos and young seedlings. Plant Physiol 151:306-322

Schultz CJ, GM Coruzzi (1995). The aspartate aminotransferase gene family of Arabidopsis encodes isoenzymes localized to three distinct subcellular compartments. Plant J 7:61-75 
Jin et al. / Intl J Agric Biol, Vol 25, No 4, 2021

Shelp BJ (2012). Does long-distance GABA signaling via the phloem really occur?. Botany 90:897-900

Shelp BJ, AW Bown, MD McLean (1999). Metabolism and functions of gamma-aminobutyric acid. Trends Plant Sci 4:446-452

Steward F (1949). $\gamma$-Aminobutyric acid: A constituent of the potato tuber. Science 110:439-440

Takahashi Y, T Sasanuma, T Abe (2013). Accumulation of gammaaminobutyrate (GABA) caused by heat-drying and expression of related genes in immature vegetable soybean (edamame). Breed Sci 63:205-210

Van Cauwenberghe OR, BJ Shelp (1999). Biochemical characterization of partially purified gaba: Pyruvate transaminase from Nicotiana tabacum. Phytochemistry 52:575-581

Wang H, LL Xiang, FH Zhang (2013). Function and application in food industry of $\gamma$-aminobutyric acid. Food Indus 34:186-189

Xu JG, QP Hu (2014). Changes in $\gamma$-aminobutyric acid content and related enzyme activities in Jindou 25 soybean (Glycine max L.) seeds during germination. LWT-Food Sci Technol 55:341-346
Yang T, K Ming, TW Zeng, XY Pu, SM Yang, J Du (2015). Difference analysis of functional components of whole grains between hull-less barley and normal barley. Southwest Chin J Agric Sci 6:2360

Zhang H, HY Yao, YR Jiang (2002). Development of the health food enriched with $\gamma$-Aminobutyric acid (GABA). Food Ferment Indus 9:69-72

Zhang YD, XW Zhou (2014). Analysis of the $\beta$-glucan content changes in germination process of highland barley kernels. Chin Agric Sci Bull 30:294-298

Zheng YM (2006). Study of germinated brown rice on its nutritional property and physiological function of gamma amino-butyric acid accumulation therein. Huazhong Agric Univ Animal Genetics Breeding Reprod Science. Wuhan, China

Zhou L, BB Shen, SY Bai, X Liu, L Jiang, HQ Zai, JM Wan (2015). RNA interference of OsGABA-T1Gene expression induced GABA accumula-tion in rice grain. Acta Agron Sin 41:1305-1312

Zhou ZW, ZM Liu, XW Zhou (2018). Research progress of highland barley processed products. Sci Technol Cereal Oils Foods 26:11-16 